\title{
OBTENCION DE PULPA KRAFT DE DESECHOS Y ASTILLAS COMERCIALES DE EUCALIPTO
}

\author{
Sandra K. Rodríguez S. (*) \\ Marco H. Torres U. (**)
}

\section{RESUMEN}

El objetivo del trabajo consiste en evaluar la aptitud pulpable de algunos tipos de desechos de eucalipto comparándolos con astillas comerciales o industriales del mismo.

Se usaron los desechos provenientes de industrias de la zona consistentes en chapas de eucalipto y despuntes de aserraderos.

Se realizaron pulpajes a distintas condiciones de cocción variando el factor $\mathrm{H}$, para 10 cual se varió el tiempo a temperatura, manteniendo el resto de las variables constantes. Las pulpas crudas se refinaron para posteriormente determinar sus propiedades fisicomecánicas.

Las pulpas resultaron con rendimientos clasificados entre 48.2 y $56.8 \%$ e índices Kappa entre 11 y 30. Las resistencias fisicomecánicas de las pulpas son aceptables para la especie, tanto con astillas comerciales como de desecho.

Palabras claves: Pulpaje kraft, Eucalyptus globulus. Desechos. Análisis quimico. Astillas. Propiedades de las pulpas.

\section{ABSTRACT}

The objective of this work is to compare pulping results among commercial Eucalyptus globulus chips and chips made of waste materials of veneers and sawnwood production.

Different pulping processes were tryed changing the relationship between time and temperature of testing. Raw pulps were refined to determine physical and mechanical properties

The results indicate classified yields on the range of $48,2 \%$ and $56,8 \%$, and Kappa indexes ranging from 11 to 30 . The physical and mechanical strength of the pulps are acceptable to the species, both from commercial and waste materials.

Keywords: Kraft pulping. Eucalyptus globulus. Waste. Chemical analysis. Chips. Pulp properties. 


\section{INTRODUCCION}

El uso de fibra corta, especialmente para la fabricación de papeles en que se requieren buena formación y características superficiales, como es el caso de papeles finos y computacionales, está cobrando gran importancia (Young, 1988). Por esta razón en los últimos años el eucalipto se ha convertido en una materia prima importante en el país y también a nivel mundial (Poblete, Foelkel et al.,1987; Foelkel, 1987; Sidaway, 1988; Zobel, 1988).

Las pulpas de eucalipto son ahora componentes comunes del abastecimiento y varian entre el 20 y $100 \%$ del suministro total. Esta trayectoria puede haber sido iniciada a causa de menores costos, pero estas pulpas están ahora siendo usadas principalmente por las excelentes propiedades que ellas imparten.

Entre los atributos de las pulpas de madera de eucalipto (Eucalyptus globulus) se encuentran volumen, suavidad, flexibilidad, buena formación y excelente opacidad y porosidad. Las pulpas de eucalipto son particularmente deseables para impresión y escritura y papeles tissue. Estas también tienden a tener un menor contenido de finos que otras pulpas de latifoliadas, que resultan en menor drenaje y más rápido secado (Zobel, 1988; Williams, 1988).

El crecimiento de la industria celulósica, está ejerciendo presión sobre los industriales para el desarrollo de nuevas fuentes de fibras, sean estas de madera u otro tipo. Es así como la tendencia actual en los paises productores de pulpa química está dirigida al empleo de desechos como materia prima, todo esto con el objeto de mejorar el rendimiento económico de bosques y aserraderos y disminuir el gran volumen de desechos producidos en diversos procesos de conversión de madera.

El problema que se aborda en este estudio se refiere a la utilización de desechos de eucalipto provenientes de fábricas de contrachapados y aserraderos, en la producción de pulpa kraft.

Como las características de los diferentes materiales son distintas, es imprescindible conocer la variación que se produce en la calidad de la pasta química, al procesar astillas de desechos de foliado, despuntes de aserraderos y astillas que resultan de la industrialización de la madera de plantaciones de eucalipto (Eucalyptus globulus).

Dado que el éxito de las pulpas de eucalipto se debe en parte a la flexibilidad del proceso kraft para adaptarse a las características de la madera (Fernandez, 1988; Higgins, 1988), en el presente estudio se hace una comparación de las pulpas obtenidas con las diferentes materias primas (astillas y desechos), usando un proceso de uso actual en Chile como lo es el del sulfato. 


\section{MATERIAL Y METODO}

\section{Preparación y Caracterización de la Materia Prima}

Se recolectaron desechos de foliado y aserradero de las fábricas laminadoras MASISA e INFODEMA, agrupándose los desechos del mismo tipo.

Las astillas comerciales o de exportación de eucalipto fueron donadas para el presente estudio por la Compañía Chilena de Astillas.

Las astillas de desechos, tanto de chapas como de despuntes se fabricaron manualmente. Las primeras con dimensiones aproximadas de $2,5 \mathrm{~cm}$ de largo y $0,5-0,8 \mathrm{~mm}$ de espesor, dado por el espesor de la chapa, y las últimas con $2,5 \mathrm{~cm}$ de largo y $4 \mathrm{~mm}$ de espesor.

Los tres tipos de astillas ( chapas, despuntes y exportación ) se clasificaron en un harnero vibratorio entre $7 / 8$ y $3 / 16$ " y en seguida se almacenaron para su posterior procesamiento.

Se determinó densidad de la madera y composición química usando las siguientes normas:

$\begin{array}{ll}\text { Holocelulosa } & \text { : Método de Poljak } \\ \text { Lignina } & \text { TAPPI T 222-om-88 } \\ \text { Etanol-Tolueno } & : \text { TAPPI T 5-om-88 } \\ \text { Soda } & \text { TAPPI T 212-om-88 } \\ \text { Cenizas } & \text { TAPPI T 15-om-88 } \\ \text { Agua fría } & \text { TAPPI T 207-om-88 } \\ \text { Agua caliente } & : \text { TAPPI T 207-om-88 }\end{array}$

\section{Ensayos de Pulpaje}

Los pulpajes se efectuaron en un digestor MK System de 6,5 litros, con recirculación forzada de licor y calefacción indirecta.

Las condiciones de cocción usadas, que fueron determinadas en base a antecedentes bibliográficos disponibles para la especie (Melo et al., 1982; Melo et al., 1991) y a trabajos realizados con anterioridad, se describen a continuación: 
Alcali activo (como $\mathrm{Na}_{2} \mathrm{O}$ ),

(\% bms)

17

Sulfidez

Temperatura máxima

Tiempo hasta temperatura

Tiempo a temperatura max.

Razón licor/madera

(\% bms)

$\left({ }^{\circ} \mathrm{C}\right)$

(min)

(min)
20

160

100

20-80

$4,5 / 1$

Los valores de factor $\mathrm{H}$ para las combinaciones de tiempo - temperatura se dan a continuación en el Cuadro №1.

\section{Cuadro $N^{\circ} 1$}

RELACION FACTOR H Y TIEMPO - TEMPERATURA

\begin{tabular}{|ccc|c|}
\hline $\begin{array}{c}\text { Tiempo } \\
(\mathrm{min})\end{array}$ & - & $\begin{array}{c}\text { Temperatura } \\
\left({ }^{\circ} \mathrm{C}\right)\end{array}$ & Factor $\mathrm{H}$ \\
\hline 20 & - & 160 & 225 \\
40 & - & 160 & 358 \\
60 & - & 160 & 490 \\
80 & - & 160 & 623 \\
\hline
\end{tabular}

Como respuesta de los pulpajes se midieron las siguientes características en la pulpa y licor, según las normas que se indican:

Rendimiento clasificado

Rechazo

Lignina residual o índice Kappa: TAPPI T 236-cm-85

Alcali residual

: TAPPI T 625-cm-88

Sólidos totales

: TAPPI T 650-pm-84

\section{Evaluación de las Propiedades Fisicomecánicas de las Pulpas}

Las pulpas se refinaron en una batidora Valley de acuerdo con la norma TAPPI T 200-om-85. A continuación se midieron las siguientes propiedades en hojas de ensayo de $60 \mathrm{~g} / \mathrm{m}^{2}$, fabricadas con pulpas a diferentes grados de refinación:
Densidad $\left(\mathrm{g} / \mathrm{cm}^{3}\right)$
: TAPPI T 220-om-88
Longitud de ruptura $(\mathrm{km})$ : TAPPI T 404-om-87
Factor de rasgado
TAPPI T 403-om-85
Factor de explosión
: TAPPI T 414-om 88 


\section{RESULTADOS Y DISCUSION}

\section{Características de la Madera}

La distribución de tamaño de las astillas seleccionadas se muestra en el Cuadro $\mathrm{N}^{\circ} 2$.

\section{Cuadro №2}

\section{DISTRIBUCION DE TAMAÑO ASTILLAS}

(\% PESO)

\begin{tabular}{|c|c|c|c|}
\hline \multirow{2}{*}{$\begin{array}{c}\text { Diámetro Malla } \\
\text { Retención } \\
\text { (“) }\end{array}$} & Tipo de Astilla \\
\cline { 2 - 4 } & Exportación & Chapas & Despuntes \\
\hline $11 / 4 \cdot 7 / 8$ & 57,3 & 24,5 & 61,7 \\
$7 / 8-5 / 8$ & 24,4 & 34,4 & 34,2 \\
$5 / 8-3 / 8$ & 15,9 & 32,7 & 3,8 \\
$3 / 8-3 / 16$ & 2,9 & 8,4 & 0,3 \\
\hline
\end{tabular}

La clasificación del material astillado (Cuadro №2) muestra una distribución de tamaños variable entre las distintas clases de astillas, lo cual se debe a la distinta forma de fabricación de éstas. Las características químicas y la densidad de la madera se detallan en el Cuadro №3.

\section{Cuadro $N^{\circ} 3$}

CARACTERISTICAS QUIMICAS DE LA MADERA EN PORCENTAJE

\section{BASE MADERA SECA}

\begin{tabular}{|l|c|c|c|}
\hline \multirow{2}{*}{ Análisis Quimico } & \multicolumn{3}{|c|}{ Tipo de Astilla } \\
\cline { 2 - 4 } & Exportación & Chapas & Despuntes \\
\hline Holocelulosa & 73.8 & 73.3 & 70.2 \\
Lignina & 22.6 & 20.8 & 26.7 \\
Solubles en: & & & \\
Etanol-tolueno & 4.3 & 6.4 & 5.8 \\
Agua caliente & 5.6 & 7.7 & 6.9 \\
Agua fria & 2.9 & 3.8 & 3.0 \\
Soda 1\% & 16.2 & 17.8 & 20.2 \\
Cenizas & 0.4 & 0.2 & 0.1 \\
Densidad $\left(\mathrm{g}^{\mathrm{cm}} \mathrm{cm}^{3}\right)$ & 0.61 & 0.51 & 0.65 \\
\hline
\end{tabular}


La densidad básica de las astillas cae en el margen de densidad media para la especie $\left(0,5-0, .6 \mathrm{~g} / \mathrm{cm}^{3}\right)$ (Melo et al., 1981; Poblete , Inzunza et al., 1987). Para las astillas de chapas la densidad resulta inferior.

Las características químicas analizadas (Cuadro №3) se determinaron en el material astillado y, por lo tanto, representan la propiedad promedio de cada tipo de astilla.

Las astillas de chapas resultaron con mayor porcentaje de extraíbles y menor de lignina.

El valor de extraíbles solubles en etanol-tolueno de las distintas astillas es alto $(5,6-7,7)$ comparado con los valores obtenidos por Melo en diversos trabajos (0,99 - 2,6) (Melo et al., 1981; Paz, Melo et al., 1987; Melo et al., 1991).

El contenido de lignina más alto se obtiene en astillas de despuntes siendo éste de $26,7 \%$.

\section{Ensayos de Pulpaje}

Las características de las pulpas; rendimiento clasificado, rechazo, rendimiento total e índice Kappa, se presentan en el Cuadro $\mathrm{N}^{\circ} 4$.

Cuadro $\mathrm{N}^{\circ} 4$.

Resultados de pulpaje

\begin{tabular}{|c|c|c|c|c|c|c|c|c|c|c|}
\hline $\begin{array}{l}\text { Tpo de } \\
\text { Astila }\end{array}$ & $\begin{array}{l}\text { Pupere } \\
\text { (N9) }\end{array}$ & $\begin{array}{c}\text { Factor } \\
\text { H }\end{array}$ & $\begin{array}{l}\text { Rendimento } \\
\text { Clasifcado } \\
\text { (Soms) }\end{array}$ & $\begin{array}{l}\text { Rechazo } \\
\text { (Socms) }\end{array}$ & $\begin{array}{l}\text { Rendimento } \\
\text { Todal } \\
\text { (Nobms) }\end{array}$ & $\begin{array}{l}\text { Indice } \\
\text { Kappa }\end{array}$ & $\begin{array}{c}\text { Rendimiento } \\
\text { Volumethco } \\
\left(\mathrm{Kg} g \mathrm{~m}^{3}\right)\end{array}$ & $\begin{array}{l}\text { Solidos } \\
\text { Totales } \\
\text { ("sov) }\end{array}$ & $\begin{array}{l}\text { Alcal } \\
\text { Ressoual } \\
\text { (gt) }\end{array}$ & $\begin{array}{c}\text { Alcal } \\
\text { Consumido } \\
(\%)\end{array}$ \\
\hline EXPORTACION & $\begin{array}{l}1 \\
2 \\
3 \\
4\end{array}$ & $\begin{array}{l}623 \\
490 \\
358 \\
225\end{array}$ & $\begin{array}{l}51.9 \\
51.6 \\
51.9 \\
48.2\end{array}$ & $\begin{array}{l}0.5 \\
1.4 \\
3.2 \\
8.6\end{array}$ & $\begin{array}{l}52.4 \\
53.0 \\
55.1 \\
56.8\end{array}$ & $\begin{array}{l}15 \\
16 \\
22 \\
30\end{array}$ & $\begin{array}{l}316 \\
315 \\
316 \\
294\end{array}$ & $\begin{array}{l}16.6 \\
16.4 \\
14.9 \\
14.9\end{array}$ & $\begin{array}{r}7.6 \\
8.5 \\
9.0 \\
10.4\end{array}$ & $\begin{array}{l}13.6 \\
13.2 \\
12.9 \\
12.3\end{array}$ \\
\hline CHAPAS & $\begin{array}{l}5 \\
6 \\
7 \\
8\end{array}$ & $\begin{array}{l}623 \\
490 \\
358 \\
225\end{array}$ & $\begin{array}{l}54.2 \\
54.6 \\
55.5 \\
56.8\end{array}$ & $\begin{array}{l}0.0 \\
0.1 \\
0.3 \\
1.4\end{array}$ & $\begin{array}{l}54.2 \\
54.7 \\
55.8 \\
58.2\end{array}$ & $\begin{array}{l}11 \\
12 \\
15 \\
26\end{array}$ & $\begin{array}{l}279 \\
281 \\
285 \\
292\end{array}$ & $\begin{array}{l}17.9 \\
16.1 \\
16.8 \\
15.0\end{array}$ & $\begin{array}{l}44 \\
5.6 \\
6.2 \\
67\end{array}$ & $\begin{array}{l}15.1 \\
14.5 \\
14.2 \\
14.0\end{array}$ \\
\hline DESPUNTES & $\begin{array}{r}9 \\
10 \\
11 \\
12\end{array}$ & $\begin{array}{l}623 \\
490 \\
358 \\
225\end{array}$ & $\begin{array}{l}50.7 \\
51.7 \\
52.6 \\
51.2\end{array}$ & $\begin{array}{l}0.6 \\
0.9 \\
12 \\
3.2\end{array}$ & $\begin{array}{l}51.3 \\
52.6 \\
53.8 \\
54.4\end{array}$ & $\begin{array}{l}15 \\
15 \\
21 \\
29\end{array}$ & $\begin{array}{l}330 \\
336 \\
342 \\
333\end{array}$ & $\begin{array}{l}17.2 \\
16.4 \\
15.5 \\
14.7\end{array}$ & $\begin{array}{l}3.7 \\
44 \\
56 \\
6.3\end{array}$ & $\begin{array}{l}15.3 \\
15.0 \\
14.5 \\
14.2\end{array}$ \\
\hline
\end{tabular}


Los ensayos de pulpaje aplicando condiciones similares en cuanto a carga de reactivos y temperatura máxima, con tiempo de reposo variable, permiten alcanzar índices Kappa entre 11 y 30 . Con astillas de chapas se obtienen los menores índices Kappa, siendo estos entre 11 y 26.

Los rendimientos son altos, fluctuando en el margen entre 48.2 y 56.8 , en términos de rendimiento aceptados para la especie. No se observa gran diferencia en rendimiento entre astillas de exportación y despuntes, pero sí entre éstas y astillas de chapas. Con las últimas se alcanzaron los mayores rendimientos clasifica$\operatorname{dos}(54,2$ y $56,8 \%)$.

Comparativamente con astillas de exportación y despuntes se obtuvieron los mayores índices Kappa, con menor rendimiento clasificado que con astillas de chapas, lo cual se aprecia en la Figura $N^{\circ} 1$. La relación entre rendimiento clasificado e índice Kappa para estas ultimas es directamente proporcional.

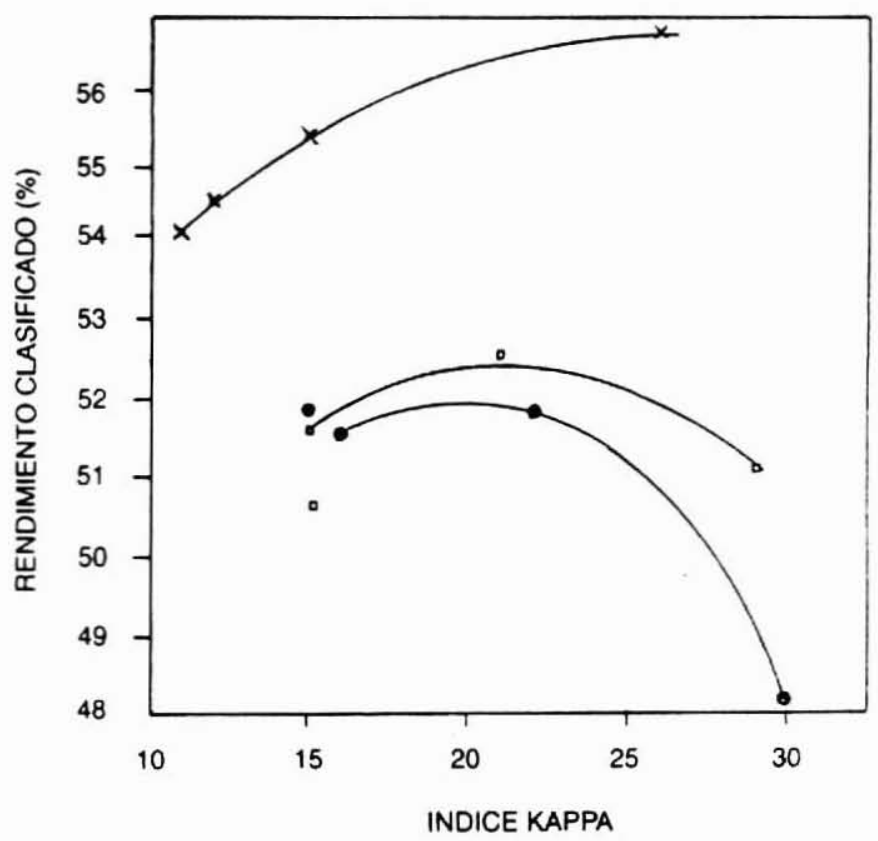

Figura $N^{\circ} 1$. RENDIMIENTO CLASIFICADO V/S INDICE KAPPA ( $\times$ CHAPAS, OASTILLAS EXPORTACION Y I DESPUNTES) 
Los diagramas obtenidos con las respuestas de los ensayos de pulpaje v/s factor $\mathrm{H}$ (Figuras $\mathrm{N}^{\circ} \mathrm{S} 2 \mathrm{a} 4$ ) permiten hacer una aproximación de las condiciones de proceso adecuadas para obtener pulpas, con las diferentes clases de astillas empleadas, que cumplirán con las condiciones simultáneas de máximo rendimiento e indice Kappa inferior a 25.

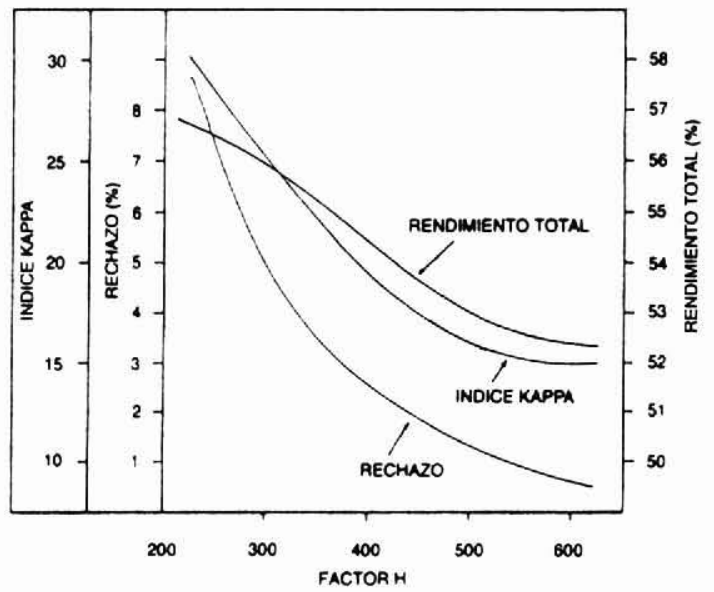

Figura No2. RESULTAdOS DE PULPAJE PARA ASTILLAS DE EXPORTACION.

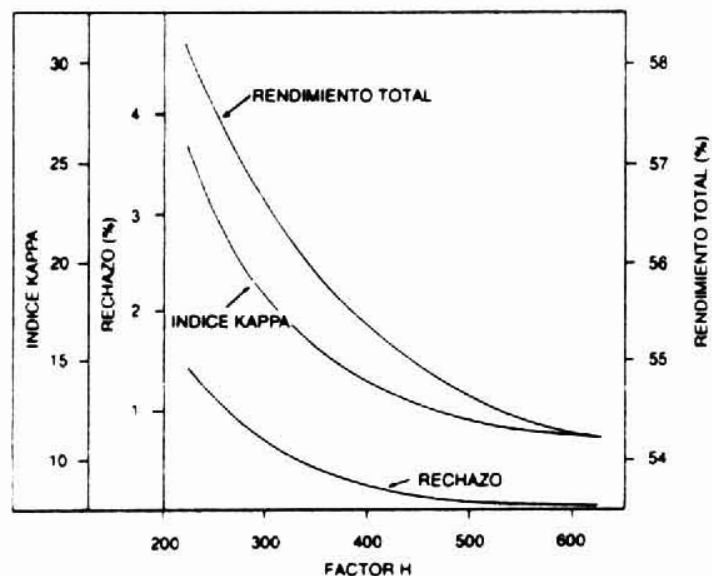

Figura No3. RESUltados de PULPAJE PARA ASTILLAS dE CHAPAS. 


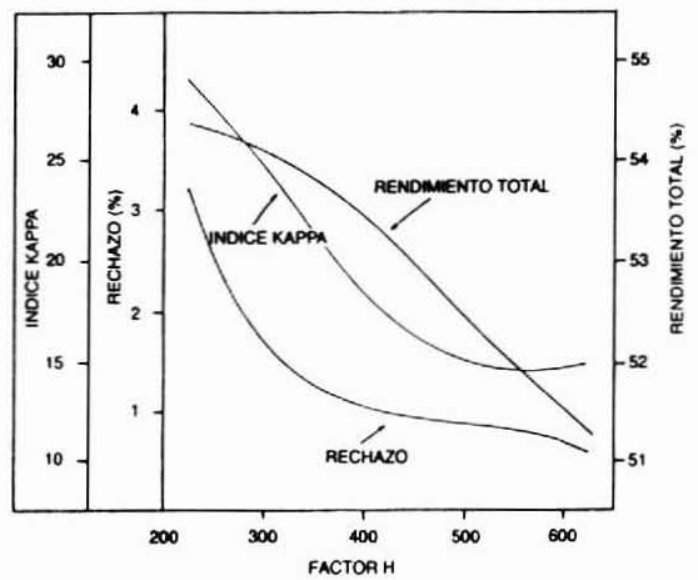

Figura No4. RESULtados de PULPAJE PARA ASTILLAS DE DESPUNTES.

Las condiciones de factor $\mathrm{H}$ y rendimiento total para obtener pulpas de índice de Kappa 20 se resumen en el Cuadro $\mathrm{N}^{\circ} 5$.

\section{Cuadro $\mathrm{N}^{\circ} 5$}

CONDICIONES DE COCCION PARA PULPAS DE I. KAPPA 20

\begin{tabular}{|l|c|c|c|}
\hline Tipo Astilla & Indice Kappa & Factor $\mathrm{H}$ & $\begin{array}{c}\text { Rendimiento } \\
\text { Total } \\
(\%)\end{array}$ \\
\hline Exportación & 20 & 400 & 54,0 \\
Chapas & 20 & 275 & 56,9 \\
Despuntes & 20 & 376 & 53,6 \\
\hline
\end{tabular}

\section{Propiedades Fisicomecánicas de las Pulpas}

Las características físicomecánicas de las pulpas se muestran en los Cuadros Nos 6 a 8. 


\section{Cuadro $\mathrm{N}^{\circ} 6$}

CARACTERISTICAS FISICOMECANICAS DE LAS PULPAS ASTILLAS DE EXPORTACION

\begin{tabular}{|c|c|c|c|c|c|c|c|}
\hline $\begin{array}{c}\text { Pulpaje } \\
\qquad N^{\circ}\end{array}$ & $\begin{array}{c}\text { Tiempo } \\
\text { de batido } \\
\text { (min) }\end{array}$ & $\begin{array}{c}\text { Drenaje } \\
\text { ('SR) }\end{array}$ & $\begin{array}{c}\text { Densidad } \\
\left(\mathrm{g} / \mathrm{cm}^{3}\right)\end{array}$ & $\begin{array}{c}\text { Longitud } \\
\text { Ruptura } \\
\text { (km) }\end{array}$ & $\begin{array}{c}\text { Factor } \\
\text { Explosión }\end{array}$ & $\begin{array}{c}\text { Factor } \\
\text { Rasgado }\end{array}$ & $\begin{array}{c}\text { Blancura } \\
\text { (\%) }\end{array}$ \\
\hline 1 & $\begin{array}{r}0 \\
5 \\
17 \\
29 \\
36\end{array}$ & $\begin{array}{l}16 \\
18 \\
25 \\
35 \\
45\end{array}$ & $\begin{array}{l}0,44 \\
0,47 \\
0,59 \\
0,67 \\
0,78\end{array}$ & $\begin{array}{r}2,4 \\
3,6 \\
6,4 \\
8,8 \\
10,0\end{array}$ & $\begin{array}{l}13 \\
23 \\
44 \\
65 \\
74\end{array}$ & $\begin{array}{r}43 \\
59 \\
93 \\
102 \\
103\end{array}$ & $\begin{array}{l}40 \\
39 \\
37 \\
36 \\
35\end{array}$ \\
\hline 2 & $\begin{array}{r}0 \\
5 \\
23 \\
35 \\
45\end{array}$ & $\begin{array}{l}15 \\
16 \\
25 \\
35 \\
45\end{array}$ & $\begin{array}{l}0,40 \\
0,45 \\
0,63 \\
0,71 \\
0,76\end{array}$ & $\begin{array}{l}1,7 \\
3,9 \\
8,0 \\
9,5 \\
9,9\end{array}$ & $\begin{array}{l}14 \\
20 \\
50 \\
69 \\
78\end{array}$ & $\begin{array}{l}32 \\
51 \\
87 \\
92 \\
94\end{array}$ & $\begin{array}{l}40 \\
39 \\
37 \\
36 \\
33\end{array}$ \\
\hline 3 & $\begin{array}{r}0 \\
5 \\
21 \\
37 \\
43\end{array}$ & $\begin{array}{l}15 \\
18 \\
25 \\
35 \\
45\end{array}$ & $\begin{array}{l}0,43 \\
0,45 \\
0,58 \\
0,73 \\
0,77\end{array}$ & $\begin{array}{r}3,0 \\
3,7 \\
7,2 \\
9,6 \\
10,5\end{array}$ & $\begin{array}{l}14 \\
23 \\
54 \\
68 \\
74\end{array}$ & $\begin{array}{l}47 \\
65 \\
90 \\
98 \\
97\end{array}$ & $\begin{array}{l}39 \\
38 \\
38 \\
35 \\
35\end{array}$ \\
\hline 4 & $\begin{array}{r}0 \\
5 \\
25 \\
37 \\
44\end{array}$ & $\begin{array}{l}13 \\
15 \\
25 \\
35 \\
45\end{array}$ & $\begin{array}{l}0,38 \\
0,43 \\
0,63 \\
0,72 \\
0,77\end{array}$ & $\begin{array}{r}1,9 \\
3,1 \\
7,6 \\
9,5 \\
10,4\end{array}$ & $\begin{array}{r}8 \\
15 \\
50 \\
68 \\
77\end{array}$ & $\begin{array}{r}30 \\
46 \\
83 \\
96 \\
100\end{array}$ & $\begin{array}{l}39 \\
38 \\
37 \\
36 \\
34\end{array}$ \\
\hline
\end{tabular}

La resistencia mecánica de las pulpas está en el margen aceptable para la especie. Los valores máximos son más bajos para astillas de exportación comparadas con las astillas de desechos. Las pulpas obtenidas con desechos de chapas (Cuadro $\mathrm{N}^{\circ}$ 7) se caracterizan por su alto factor de rasgado (valor máximo 136) superior en todos los casos a las restantes clases de astillas.

Por otra parte las pulpas de desechos de despuntes tienen alta longitud de ruptura y factor de explosión, con valores máximos $10.7 \mathrm{~km}$ y 84 , respectivamente (Cuadro $N^{\circ} 8$ ).

La mayoría de las pulpas, a $25^{\circ} \mathrm{SR}$ sobrepasan el valor 90 para factor de rasgado, excepto la pulpa $N^{\circ} 2$ obtenida con astillas de exportación. A ese mismo valor de drenaje las pulpas alcanzan sobre $6 \mathrm{~km}$ de longitud de ruptura, cumpliendo de esta forma con las exigencias de resistencia fisicomecánica para papeles de impresión. 


\section{Cuadro $N^{\circ} 7$}

\section{CARACTERISTICAS FISICOMECANICAS DE LAS PULPAS ASTILLAS DE CHAPAS}

\begin{tabular}{|c|c|c|c|c|c|c|c|}
\hline $\begin{array}{c}\text { Pulpaje } \\
N^{\circ}\end{array}$ & $\begin{array}{c}\text { Tiempo } \\
\text { de batido } \\
\text { (min) }\end{array}$ & $\begin{array}{c}\text { Drenaje } \\
\text { ('SR) }\end{array}$ & $\begin{array}{c}\text { Densidad } \\
\left(\mathrm{g} / \mathrm{cm}^{3}\right)\end{array}$ & $\begin{array}{c}\text { Longitud } \\
\text { Ruptura } \\
\text { (km) }\end{array}$ & $\begin{array}{c}\text { Factor } \\
\text { Explosión }\end{array}$ & $\begin{array}{c}\text { Factor } \\
\text { Rasgado }\end{array}$ & $\begin{array}{c}\text { Blancura } \\
(\%)\end{array}$ \\
\hline 5 & $\begin{array}{r}0 \\
5 \\
27 \\
41 \\
46\end{array}$ & $\begin{array}{l}16 \\
17 \\
25 \\
35 \\
45\end{array}$ & $\begin{array}{l}0,41 \\
0,47 \\
0,62 \\
0,70 \\
0,73\end{array}$ & $\begin{array}{l}2,5 \\
3,8 \\
7,2 \\
9,0 \\
9,4\end{array}$ & $\begin{array}{l}18 \\
28 \\
57 \\
72 \\
75\end{array}$ & $\begin{array}{r}52 \\
83 \\
115 \\
127 \\
125\end{array}$ & $\begin{array}{l}37 \\
36 \\
35 \\
34 \\
34\end{array}$ \\
\hline 6 & $\begin{array}{r}0 \\
5 \\
23 \\
42 \\
47\end{array}$ & $\begin{array}{l}15 \\
17 \\
25 \\
35 \\
45\end{array}$ & $\begin{array}{l}0,43 \\
0,46 \\
0,63 \\
0,71 \\
0,74\end{array}$ & $\begin{array}{r}3,6 \\
4,6 \\
9,3 \\
10,7 \\
9,2\end{array}$ & $\begin{array}{l}17 \\
26 \\
75 \\
79 \\
64\end{array}$ & $\begin{array}{r}57 \\
69 \\
114 \\
122 \\
121\end{array}$ & $\begin{array}{l}38 \\
37 \\
36 \\
34 \\
31\end{array}$ \\
\hline 7 & $\begin{array}{r}0 \\
5 \\
25 \\
40 \\
47\end{array}$ & $\begin{array}{l}16 \\
17 \\
25 \\
35 \\
45\end{array}$ & $\begin{array}{l}0,42 \\
0,45 \\
0,60 \\
0,68 \\
0,71\end{array}$ & $\begin{array}{r}3,0 \\
4,5 \\
8,1 \\
9,6 \\
10,3\end{array}$ & $\begin{array}{l}17 \\
26 \\
61 \\
74 \\
78\end{array}$ & $\begin{array}{r}61 \\
77 \\
117 \\
128 \\
128\end{array}$ & $\begin{array}{l}36 \\
36 \\
35 \\
35 \\
34\end{array}$ \\
\hline 8 & $\begin{array}{r}0 \\
5 \\
28 \\
42 \\
50\end{array}$ & $\begin{array}{l}14 \\
15 \\
25 \\
35 \\
45\end{array}$ & $\begin{array}{l}0,40 \\
0,42 \\
0,60 \\
0,70 \\
0,74\end{array}$ & $\begin{array}{l}1,9 \\
2,6 \\
7,2 \\
8,9 \\
9,3\end{array}$ & $\begin{array}{l}12 \\
18 \\
52 \\
72 \\
80\end{array}$ & $\begin{array}{r}58 \\
68 \\
117 \\
132 \\
136\end{array}$ & $\begin{array}{l}35 \\
34 \\
34 \\
33 \\
32\end{array}$ \\
\hline
\end{tabular}


Cuadro $\mathrm{N}^{\circ} 8$

CARACTERISTICAS FISICOMECANICAS DE LAS PULPAS ASTILLAS DE DESPUNTES

\begin{tabular}{|c|c|c|c|c|c|c|c|}
\hline $\begin{array}{c}\text { Pulpaje } \\
N^{\circ}\end{array}$ & $\begin{array}{c}\text { Tiempo } \\
\text { de batido } \\
\text { (min) }\end{array}$ & $\begin{array}{c}\text { Drenaje } \\
\text { ('SR) }\end{array}$ & $\begin{array}{l}\text { Densidad } \\
\left(\mathrm{g} / \mathrm{cm}^{3}\right)\end{array}$ & $\begin{array}{c}\text { Longitud } \\
\text { Ruptura } \\
\text { (Km) }\end{array}$ & $\begin{array}{c}\text { Factor } \\
\text { Explosión }\end{array}$ & $\begin{array}{c}\text { Factor } \\
\text { Rasgado }\end{array}$ & $\begin{array}{c}\text { Blancura } \\
\text { (\%) }\end{array}$ \\
\hline 9 & $\begin{array}{r}0 \\
5 \\
23 \\
38 \\
45\end{array}$ & $\begin{array}{l}17 \\
19 \\
25 \\
35 \\
45\end{array}$ & $\begin{array}{l}0,47 \\
0,50 \\
0,68 \\
0,78 \\
0,81\end{array}$ & $\begin{array}{l}3,1 \\
3,6 \\
6,4 \\
9,1 \\
9,8\end{array}$ & $\begin{array}{l}19 \\
27 \\
53 \\
76 \\
81\end{array}$ & $\begin{array}{r}59 \\
76 \\
103 \\
116 \\
118\end{array}$ & $\begin{array}{l}38 \\
37 \\
35 \\
34 \\
33\end{array}$ \\
\hline 10 & $\begin{array}{r}0 \\
5 \\
24 \\
39 \\
45\end{array}$ & $\begin{array}{l}17 \\
19 \\
25 \\
35 \\
45\end{array}$ & $\begin{array}{l}0,46 \\
0,53 \\
0,69 \\
0,79 \\
0,83\end{array}$ & $\begin{array}{r}3,4 \\
3,9 \\
8,0 \\
10,5 \\
11,2\end{array}$ & $\begin{array}{l}22 \\
31 \\
57 \\
73 \\
81\end{array}$ & $\begin{array}{r}53 \\
66 \\
100 \\
114 \\
119\end{array}$ & $\begin{array}{l}38 \\
35 \\
33 \\
33 \\
30\end{array}$ \\
\hline 11 & $\begin{array}{r}0 \\
5 \\
25 \\
39 \\
45\end{array}$ & $\begin{array}{l}17 \\
18 \\
25 \\
35 \\
45\end{array}$ & $\begin{array}{l}0,49 \\
0,56 \\
0,70 \\
0,79 \\
0,83\end{array}$ & $\begin{array}{l}2,6 \\
5,0 \\
6,9 \\
9,0 \\
9,9\end{array}$ & $\begin{array}{l}22 \\
28 \\
55 \\
75 \\
84\end{array}$ & $\begin{array}{r}60 \\
71 \\
112 \\
123 \\
120\end{array}$ & $\begin{array}{l}36 \\
34 \\
33 \\
32 \\
30\end{array}$ \\
\hline 12 & $\begin{array}{r}0 \\
5 \\
25 \\
43 \\
52\end{array}$ & $\begin{array}{l}15 \\
16 \\
25 \\
35 \\
45\end{array}$ & $\begin{array}{l}0,45 \\
0,50 \\
0,67 \\
0,77 \\
0,82\end{array}$ & $\begin{array}{r}2,4 \\
3,5 \\
7,5 \\
9,6 \\
10,7\end{array}$ & $\begin{array}{l}16 \\
25 \\
56 \\
74 \\
80\end{array}$ & $\begin{array}{r}55 \\
68 \\
97 \\
110 \\
109\end{array}$ & $\begin{array}{l}37 \\
36 \\
35 \\
33 \\
31\end{array}$ \\
\hline
\end{tabular}

\section{CONCLUSIONES}

Las características químicas de la madera se encuentran dentro del margen conocido para la especie Eucalyptus globulus, a excepción del alto contenido de extraíbles solubles en etanol-tolueno que se observó en los tres tipos de astillas estudiadas.

Se obtuvieron pulpas con rendimientos clasificados e índices Kappa aceptables para la especie. Resultando mayormente deslignificadas las pulpas provenientes de astillas de chapas, para igual factor $\mathrm{H}$.

Las resistencias fisicomecánicas de las pulpas resultan aceptables para la especie, tanto para astillas de exportación como de desechos, e incluso estas últimas 
superan a las primeras.

Los tres tipos de astillas y en especial los obtenidos de desechos presentan buenas aptitudes para ser usadas en la obtención de pulpa kraft para la fabricación de papeles.

\section{REFERENCIAS BIBLIOGRAFICAS}

Fernandez, A.,1988. Latin American Notes. Tappi Journal 71(12):25-26.

Foelkel, C.,1987. "Los eucaliptos en la fabricación de celulosa y papel en Brasil". Celulosa y Papel 3(5):9-12.

Higgins, H. G.,1988. Letter from Australia. Tappi Journal 71(12):20-22.

Melo, R.; Paz, J.; Solis, A,; Carrasco, V.; Rojas, M,; Rivera, G.; y Barriga A., 1981, "Evaluación de Recursos Fibrosos en la Subregión Andina". II Parte: “Los Eucaliptos en Chile". Laboratorio de Productos Forestales. Instituto de Investigaciones Tecnológicas. Facultad de Ingenieria. Universidad de Concepción 37p.

Melo, R.; Rojas, M. y Fuentes, L.,1982. Obtención de Pulpas Semi químicas de eucalipto (Eucalyptus spp.). Laboratorio de Productos Forestales, Facultad de Ingenieria, Universidad de Concepción.55p.

Melo, R.; Paz, J.; Solis, A. y Carrasco, V., 1991. Ensayos de pulpaje y blanqueo de madera de eucaliptos (Eucalyptus spp.). Celulosa y papel 7(1):10-19.

Paz, J.; Melo, R. et al.,1987. "Nuevas especies en la producción de celulosa". Celulosa y Papel 3(1):13-15.

Poblete, A,; Foelkel, C.; Alamo, S. y Rojas, P.,1987. Mesa Redonda I. Eucaliptus: ¿Fibra del Futuro?. Celulosa y Papel 3(4):14-15.

Poblete, H.; Inzunza, L. y Fernandez, A., 1987. Determinación del peso específico y densidad anhidra en madera de Eucalyptus spp. para exportaciones de chips. Informe de Convenio $\mathrm{N}^{\circ}{ }^{133}$. Inversiones Forestales CCA - Universidad Austral de Chile.

Sidaway, S.,1988. The availability and use of Eucalyptus pulps. Tappi Journal 71(12):47 -51 .

Williams, W. C.,1988. International Scene. Tappi Journal 71(12):24.

Young, J., 1988. Bleaching said to be key to CTMP from Eucalyptus. Tappi Journal 71(12):11,14.

Zobel, B., 1988. Eucalyptus in the forestry industry. Tappi Journal 71(12):42-46. 